Cite this: J. Mater. Chem. A, 2013, 1, 2050

Received 30th August 2012 Accepted 27th November 2012

DOI: $10.1039 / c 2 t a 00131 d$

www.rsc.org/MaterialsA

\title{
Comparison of functionalized carbon nanofibers and multi-walled carbon nanotubes as supports for Fe-Co nanoparticlest
}

\author{
Deborah Vidick, ${ }^{a}$ Marcus Herlitschke, ${ }^{\text {bc }}$ Claude Poleunis, ${ }^{d}$ Arnaud Delcorte, ${ }^{d}$ \\ Raphael P. Hermann, ${ }^{\text {bc }}$ Michel Devillers ${ }^{a}$ and Sophie Hermans ${ }^{* a}$
}

\begin{abstract}
Multi-walled carbon nanotubes (MWCNT) and nanofibers (CNF) have been functionalized at their surfaces with chelating phosphine $\left(\mathrm{PPh}_{2}\right)$ and ammonium $\left(\mathrm{NMe}_{3}{ }^{+}\right.$) groups, as anchoring sites for metal complexes. The surface functionalization has been monitored by XPS, elemental analyses, $\mathrm{N}_{2}$ physisorption and/or SEM surface morphology analysis at each step. Bimetallic Fe-Co nanoparticles from two different starting cluster complexes, $\left[\mathrm{HFeCO}_{3}(\mathrm{CO})_{12}\right](\mathbf{1})$ and $\left(\mathrm{NEt}_{4}\right)\left[\mathrm{FeCO}_{3}(\mathrm{CO})_{12}\right](2)$, have been deposited onto the surfaces of the functionalized MWCNT and CNF as well as their pristine forms for comparison. The samples have been fully characterized before and after thermal treatment. The obtained nanoparticles were shown by TEM to be better dispersed and of smaller size on functionalized than on pristine supports. Magnetic characterization revealed blocked superparamagnetic Fe-Co nanoparticles together with paramagnetic ions on CNF as well as MWCNT.
\end{abstract}

\section{Introduction}

Carbon nanotubes (CNT) and nanofibers (CNF) are known for their numerous applications in optical and electronic devices, sensors, energy storage, reinforcement for composites and as supports for heterogeneous catalysis. The growing interest in carbon materials in catalysis is due to their specific characteristics: (i) acid/basic resistance, (ii) high surface area (iii) modifiable chemical and physical properties of the surface and (iv) easy recovery of precious metals by combustion. ${ }^{1}$ Given that CNT and CNF present relatively inert surfaces, pre-treatments are needed to achieve desirable interaction with metals. Therefore, surface functionalization is essential. Surface oxidation with nitric acid or a mixture of $\mathrm{HNO}_{3}-\mathrm{H}_{2} \mathrm{SO}_{4}$ is the most common method and mainly generates carboxylic acid groups, which, in turn, can react with different types of molecules with desired functionalities. ${ }^{2-5}$ For example, carboxylic acid groups are first acylated with thionyl chloride and then

${ }^{a}$ Institute of Condensed Matter and Nanosciences (IMCN), Université catholique de Louvain, Place Louis Pasteur, 1/3, B-1348 Louvain-la-Neuve, Belgium. E-mail: Sophie.Hermans@uclouvain.be; Fax: +32 104723 30; Tel: +32 10472810

${ }^{b} J u ̈ l i c h$ Centre for Neutron Science JCNS and Peter Grünberg Institut PGI, JARA-FIT, Forschungszentrum Jülich GmbH, D-52425 Jülich, Germany. E-mail: r.hermann@ fz-juelich.de

${ }^{c}$ Faculté des Sciences, Université de Liège, B-400o, Liège, Belgium

${ }^{d}$ Institute of Condensed Matter and Nanosciences (IMCN), Université catholique de Louvain, Croix du Sud, 1, B-1348 Louvain-la-Neuve, Belgium

$\dagger$ Electronic supplementary information (ESI) available: Tables with XPS results, thermogravimetric analyses, TEM histograms, selected XPS spectra and incorporation yields. See DOI: 10.1039/c2ta00131d react with amines to form an amide bond..$^{6,7}$ The pending arm can undergo additional modifications to lead to a wide range of surface functional groups. ${ }^{2}$ Our goal is to introduce ammonium groups or phosphines at the carbon surface as anchors for noble metal complexes. The formation of ammonium groups on inorganic materials is well known ${ }^{8,9}$ but few papers deal with the preparation of ammonium-functionalized carbon nanotubes. ${ }^{10}$ Hou et al. converted amine groups into ammonium moieties and grafted gold nanoparticles on ammonium-functionalized multiwalled carbon nanotubes by anion exchange. ${ }^{10}$ Regarding phosphine groups, only four papers reported the incorporation of phosphines on SWCNT. ${ }^{11-14}$ Hamilton $e t$ al. report the functionalization of SWCNT with (hydroxymethyl) diethylphosphine or (hydroxyphenyl) diphenylphosphine in order to coordinate FeMo complexes. ${ }^{13,14}$

The chemistry of transition metal clusters was the subject of intense investigation throughout the 1970 s and 1980 s. Over the past decade there has been a gradual resurgence in the area due to the growing interest in nanotechnology. ${ }^{15}$ Since the properties of nanoparticles are size-dependent, the ability to prepare them with well-defined and narrow size distributions is of key importance. Most metallic nanoparticle syntheses are based on the reduction of metal salts in the presence of stabilizing agents. While this approach can often be carried out using commercially available reagents, the lack of size, shape and composition control leads to severe limitations. ${ }^{15}$ Clusters are adequate precursors of nanoparticles, because of their molecular nature and solubility in most organic solvents. Mixed-metal clusters have been claimed for a long time to be suitable precursors for the preparation of nanoparticles with a 
well-defined and tuneable size and stoichiometry. ${ }^{16}$ In the case of applications in catalysis, supported catalysts derived from bimetallic clusters have been shown to exhibit more desirable behaviour than conventional catalysts. ${ }^{17}$ It was shown that thermal decomposition of supported heterometallic carbonyl clusters yields very small heterometallic particles which have the same bulk composition as the starting clusters. ${ }^{18}$ However, even when using clusters as nanoparticle precursors, the supported materials present a particle size distribution, because agglomeration cannot be totally avoided. We propose that clusters need to be anchored covalently onto the surface to overcome this drawback.

Here we report the comparative functionalization of $\mathrm{CNF}$ and CNT, in order to introduce at their surface chelating phosphine $\left(\mathrm{PPh}_{2}\right)$ or ammonium $\left(\mathrm{NMe}_{3}{ }^{+}\right)$groups. These are then used as anchors for the incorporation of metallic clusters. Indeed, it is known that transition metal clusters exchange readily their CO ligands for phosphines, which would in the $\mathrm{PPh}_{2}$ case lead to covalent grafting. The ammonium groups are justified by their cationic charges allowing electrostatic interactions to take place with charged clusters that are usually anionic. The functionalizations were realized by surface oxidation followed by addition of a diamine and formation of an amide bond. The $\mathrm{PPh}_{2}$ or $\mathrm{NMe}_{3}{ }^{+}$coordination sites were then formed by derivation of the pendant arm. In the first case, the transformation of the pending amine into bidentate phosphine was realized according to our previously published procedure. ${ }^{7}$ In the second case, a quaternization reaction was used in order to create ammonium groups on the surface. Two heterometallic clusters, $\left[\mathrm{HFeCo}_{3}(\mathrm{CO})_{12}\right]$ (1) and $\left(\mathrm{NEt}_{4}\right)\left[\mathrm{FeCo}_{3}(\mathrm{CO})_{12}\right]$ (2), as representative examples of neutral and charged clusters, were incorporated onto the carbon nanofibers and nanotubes, functionalized or not. The fate of these clusters was analyzed after incorporation and thermal treatment to yield carbonsupported bimetallic nanoparticles. The selected bimetallic combination (Fe-Co) is justified by possible applications in Fischer-Tropsch catalysis or for their magnetic properties. In the present paper, we only probed their magnetic properties.

\section{Experimental}

All manipulations were carried out under $\mathrm{N}_{2}$ using standard Schlenk techniques. The solvents were distilled or degassed before use, stored under nitrogen on molecular sieves, and the obtained products were stored under argon in a glovebox. The CNF (PR-24-XT-PS, PR-24-XT-LHT and PR-24-XT-PS-OX) were received from Applied Sciences Inc. (USA). The PS grade CNF was produced by pyrolytically stripping the as-produced fibers. The LHT grade was produced by heat-treating the fibers at $1500{ }^{\circ} \mathrm{C}$. The "XT" label means that the fibers are in the debulked form and "OX" means that the fibers have been oxidized by the manufacturer. For simplicity, PR-24-XT-PS, PR-24-XT-LHT and PR-24-XT-PS-OX are re-named as CNF-PS, CNF-LHT and CNF-OX, respectively. MWCNT (90\% C purity) were supplied by Nanocyl S.A. (Belgium). All other mentioned reactants were commercially available and used as received. $\mathrm{H}_{2} \mathrm{~N}\left(\mathrm{CH}_{2}\right)_{2} \mathrm{NH}_{2},\left(\mathrm{CH}_{2} \mathrm{O}\right)_{n}$, $\mathrm{CF}_{3} \mathrm{SO}_{3} \mathrm{CH}_{3}$ and $\mathrm{Fe}(\mathrm{CO})_{5}$ were purchased from Sigma-Aldrich;
$\mathrm{SOCl}_{2}, \mathrm{Co}_{2}(\mathrm{CO})_{8}, \mathrm{NEt}_{4} \mathrm{Br}$ from Acros Organics; $\mathrm{HPPh}_{2}$ and $\mathrm{H}_{2} \mathrm{~N}\left(\mathrm{CH}_{2}\right)_{2} \mathrm{~N}\left(\mathrm{CH}_{3}\right)_{2}$ from Fluka; $65 \mathrm{wt} \%$ nitric acid from VWR and $36 \mathrm{wt} \%$ hydrochloric acid from Fisher Scientific.

\subsection{Functionalization of carbon nanofibers}

The starting nanofibers PR-24-XT-PS-OX (re-named CNF-OX) were estimated to present $1.5 \mathrm{mmol} \mathrm{g}^{-1}$ acidic functions from the XPS O/C surface atomic ratio equal to $0.116 .{ }^{19}$

2.1.1 Addition of $\mathrm{SOCl}_{2}$ onto CNF-OX to give CNF-Cl. In a $500 \mathrm{ml}$ round-bottom flask, $4 \mathrm{~g}$ of CNF-OX were introduced and degassed overnight at $120{ }^{\circ} \mathrm{C}$. Then, $130 \mathrm{ml}$ toluene were introduced together with $20 \mathrm{ml} \mathrm{SOCl}$. The mixture was refluxed $\left(120^{\circ} \mathrm{C}\right)$ for $5 \mathrm{~h}$, filtered, and the obtained powder was extensively washed with toluene and dried under vacuum for several hours.

2.1.2 Formation of $\mathbf{C N F}-\mathbf{N R}_{2}$. In a $500 \mathrm{ml}$ round-bottom flask, $3.8 \mathrm{~g}$ of CNF-Cl were introduced together with $170 \mathrm{ml}$ toluene. Then, 1.3 equivalents of $\mathrm{NH}_{2}\left(\mathrm{CH}_{2}\right)_{2} \mathrm{NR}_{2}(7.41 \mathrm{mmol}$, $0.50 \mathrm{ml}$ for $\mathrm{R}=\mathrm{H} ; 7.41 \mathrm{mmol}, 0.81 \mathrm{ml}$ for $\mathrm{R}=\mathrm{CH}_{3}$, with respect to the number of acidic functions estimated in $3.8 \mathrm{~g}$ of oxidized $\mathrm{CNF}$ ) were added. The mixture was refluxed at $120^{\circ} \mathrm{C}$ for $4 \mathrm{~h}$ to give $\mathrm{CNF}-\mathrm{NH}_{2}$ or $\mathrm{CNF}-\mathrm{NMe}_{2}$. Finally, the solid was filtered out, extensively washed with toluene and dried under vacuum for several hours.

2.1.3 Formation of CNF-PPh $\mathrm{P}_{2}$ by reaction of $\mathrm{HPPh}_{2}$ on CNF$\mathbf{N H}_{2}$ (ref. 7) In a $100 \mathrm{ml}$ round-bottom flask, $2.34 \mathrm{ml} \mathrm{HPPh}_{2}$ (13.5 mmol, 2.5 equivalents with regard to the number of acidic functions estimated in $3.6 \mathrm{~g}$ of oxidized CNF) and $405.3 \mathrm{mg}$ $\mathrm{CH}_{2} \mathrm{O}(13.5 \mathrm{mmol})$ were introduced with $20 \mathrm{ml}$ methanol. The mixture was stirred at $70{ }^{\circ} \mathrm{C}$ for $10 \mathrm{~min}$ and then cooled down to room temperature, and then added to a suspension of $3.6 \mathrm{~g}$ of CNF- $\mathrm{NH}_{2}$ in $90 \mathrm{ml}$ methanol in a $500 \mathrm{ml}$ round-bottom flask. It was stirred for $15 \mathrm{~min}$ at room temperature and then at $70{ }^{\circ} \mathrm{C}$ for $24 \mathrm{~h}$, after addition of $112.5 \mathrm{ml}$ toluene, to give $\mathrm{CNF}-\mathrm{PPh}_{2}$. Finally, the solid was filtered out, extensively washed with methanol and dried under vacuum for several hours.

2.1.4 Formation of CNF-NMe ${ }_{3}^{+}$by addition of $\mathrm{CF}_{3} \mathrm{SO}_{3} \mathrm{CH}_{3}$ on CNF-NMe ${ }_{2}$. In a $500 \mathrm{ml}$ round-bottom flask, $3.6 \mathrm{~g}$ of CNF$\mathrm{NMe}_{2}$ were introduced with $200 \mathrm{ml}$ acetone and $1.63 \mathrm{ml}$ $\mathrm{CF}_{3} \mathrm{SO}_{3} \mathrm{CH}_{3}$ (14.4 mmol, 5 equivalents with regard to the number of acidic functions estimated in $3.6 \mathrm{~g}$ of oxidized $\mathrm{CNF}$ ) were added. The mixture was stirred at room temperature for $24 \mathrm{~h}$. The solid was recovered by filtration, extensively washed with acetone and dried under vacuum for several hours.

\subsection{Functionalization of carbon nanotubes}

2.2.1 Oxidation of MWCNT to give MWCNT-OX. In a typical experiment, $1 \mathrm{~g}$ of MWCNT was placed in a $250 \mathrm{ml}$ roundbottom flask with $100 \mathrm{ml}$ concentrated $\mathrm{HNO}_{3}$. The mixture was stirred at $110{ }^{\circ} \mathrm{C}$ for $2 \mathrm{~h}, 4 \mathrm{~h}, 8 \mathrm{~h}, 12 \mathrm{~h}, 16 \mathrm{~h}$ or $24 \mathrm{~h}$ to find the optimal oxidation conditions. Then, the solid was filtered out in each case and washed with distilled water until neutral $\mathrm{pH}$. The resulting powders were dried under vacuum at $70{ }^{\circ} \mathrm{C}$ for $16 \mathrm{~h}$. The subsequent steps were realized with the optimal solid named MWCNT-OX, which had been reacted for only $2 \mathrm{~h}$. It was 
characterized by XPS $(\mathrm{O} / \mathrm{C}$ surface ratio $=0.055)$ and estimated to present $0.3 \mathrm{mmol} \mathrm{g}^{-1}$ acidic functions. ${ }^{19}$

2.2.2 Formation of MWCNT-Cl. Similar to the procedure described in 2.1.1, $900 \mathrm{mg}$ MWCNT-OX were reacted in $40 \mathrm{ml}$ toluene with $5 \mathrm{ml} \mathrm{SOCl}$.

2.2.3 Formation of MWCNT-NR $\mathbf{N}_{2}$. Similar to the procedure in 2.1.2, $800 \mathrm{mg}$ of MWCNT-Cl were reacted in $30 \mathrm{ml}$ toluene with 1.3 equivalents of $\mathrm{H}_{2} \mathrm{~N}\left(\mathrm{CH}_{2}\right)_{2} \mathrm{NR}_{2}$.

2.2.4 Formation of $\mathbf{M W C N T}-\mathbf{P P h}_{2}$. Similar to the procedure in 2.1.3, $700 \mathrm{mg}$ of MWCNT- $\mathrm{NH}_{2}$ were reacted in a mixture of methanol-toluene $(20 \mathrm{ml} / 25 \mathrm{ml})$ with 2.5 equivalents of $\mathrm{HPPh}_{2}$ and $\mathrm{CH}_{2} \mathrm{O}$.

2.2.5 Formation of MWCNT-NMe ${ }_{3}{ }^{+}$. Similar to the procedure in $2.1 .4,700 \mathrm{mg}$ of $\mathrm{MWCNT}-\mathrm{NMe}_{2}$ were reacted in $30 \mathrm{ml}$ acetone with 5 equivalents of $\mathrm{CF}_{3} \mathrm{SO}_{3} \mathrm{CH}_{3}$.

\subsection{Synthesis of bimetallic clusters}

Both clusters were prepared as previously described. ${ }^{\mathbf{2 0 2 1}}[\mathrm{HFe}-$ $\left.\mathrm{Co}_{3}(\mathrm{CO})_{12}\right]$ (1) was obtained in $50 \%$ yield. IR $\nu_{\mathrm{CO}}\left(\mathrm{CCl}_{4}\right)=2100$ (w), 2059 (s), 2050 (s), 2027 (m), 1986 (m), 1883 (s) cm ${ }^{-1}$. Anal (calc.) = C 22.43 (25.30), H $0.23(0.18) \%$. Cluster $\left(\mathrm{NEt}_{4}\right)$ $\left[\mathrm{FeCo}_{3}(\mathrm{CO})_{12}\right](2)$ was obtained in $80 \%$ yield. IR $\nu_{\mathrm{CO}}$ (acetone) $=$ 2063 (w), 2005 (s), 1970 (w), 1932 (m), 1823 (m) cm cm $^{-1}$ Anal (calc.) = C 34.17 (34.36), H 2.88 (2.88), N 1.99 (2.00)\%.

\subsection{Deposition and thermal decomposition of metal clusters on carbonaceous materials}

To aim at $5 \mathrm{wt} \%$ pure metals loading on the support media, in a typical experiment, $12.2 \mathrm{mg}$ of cluster 1 or $15 \mathrm{mg}$ of cluster 2 was stirred with $95 \mathrm{mg}$ of a carbonaceous support in a mixture of one or two solvent(s) $(1: 1$, total volume $=20 \mathrm{ml})$ at room temperature for 5 days in the dark. The solid was then filtered out, washed and dried at room temperature under vacuum. The dried sample was then placed into porcelain boats and heated under a $\mathrm{N}_{2}$ stream at $200{ }^{\circ} \mathrm{C}$ for $1 \mathrm{~h}$ for cluster 1 and at $300{ }^{\circ} \mathrm{C}$ for $1 \mathrm{~h}$ for cluster 2 (heating ramp: $100{ }^{\circ} \mathrm{C} \mathrm{h}^{-1}$ ) in a tubular oven STF 16/450 from CARBOLITE.

\subsection{Physico-chemical methods of characterization}

Infrared spectra of the clusters were recorded on a BRUKER EQUINOX 55 spectrometer with a solution cell from PERKIN ELMER. Elemental analyses $(\mathrm{C}, \mathrm{H}, \mathrm{N}, \mathrm{O}, \mathrm{Cl}, \mathrm{S}, \mathrm{P}$ or $\mathrm{F})$ were performed by MEDAC Ltd, UK.

XPS analyses were performed on a Kratos Axis Ultra spectrometer (Kratos Analytical - Manchester - UK) equipped with a monochromatized aluminium X-ray source (powered at $10 \mathrm{~mA}$ and $15 \mathrm{kV}$ ). The sample powders were pressed into small stainless steel troughs mounted on a multi-specimen holder. The pressure in the analysis chamber was about $10^{-6} \mathrm{~Pa}$. The angle between the normal to the sample surface and the direction of photoelectron collection was about $0^{\circ}$. Analyses were performed in the hybrid lens mode, the resulting analyzed area being $700 \mu \mathrm{m} \times 300 \mu \mathrm{m}$. The pass energy was set at $160 \mathrm{eV}$ for the survey scan and $40 \mathrm{eV}$ for narrow scans. In the latter conditions, the full width at half maximum (FWHM) of the $\mathrm{Ag}$ $3 \mathrm{~d}_{5 / 2}$ peak of a standard silver sample was about $0.9 \mathrm{eV}$. Charge stabilization was achieved by using the Kratos Axis device. The following sequence of spectra was recorded: survey spectrum, $\mathrm{C}$ 1s, O 1s, N 1s, Cl 2p, S 2p, P 2p (or F 1s), Co 2p and C 1s again to check for charge stability as a function of time and the absence of degradation of the sample during the analyses. The $\mathrm{C}-(\mathrm{C}, \mathrm{H})$ component of the $\mathrm{C} 1 \mathrm{~s}$ carbon peak has been fixed at $284.8 \mathrm{eV}$ to set the binding energy scale. Spectra were decomposed with the CasaXPS program (Casa Software Ltd, UK) with a Gaussian/ Lorentzian (70/30) product function and after subtraction of a linear baseline. The constraints used for decomposition of p doublets are the following: (i) area ratio $2 \mathrm{p}_{1 / 2} / 2 \mathrm{p}_{3 / 2}=0.5$ and (ii) $\mathrm{FWHM}$ ratio $=1$ and $\Delta\left(2 \mathrm{p}_{1 / 2}-2 \mathrm{p}_{3 / 2}\right)=1.6(\mathrm{Cl}), 1.18(\mathrm{~S}), 0.84$ (P), 14.97 (Co) eV. The pseudo in situ XPS measurements were carried out as described in the ESI $\dagger$ section.

Nitrogen adsorption-desorption isotherms were obtained on an ASAP 2020 instrument from MICROMERITICS at $77 \mathrm{~K}$. The sample was degassed for several hours at $200{ }^{\circ} \mathrm{C}$ before analysis. The specific surface area was calculated from the adsorption data using the Brunauer-Emmet-Teller (BET) method. The pore size distribution was obtained from the adsorption branch by using the Barrett-Joyner-Halenda (BJH) method.

Cobalt atomic absorption analyses were carried out on a PERKIN ELMER 3110 atomic absorption spectrometer. A calibration curve was realized from a Co standard solution (Fluka, $\left.1 \mathrm{~g} \mathrm{l}^{-1}\right)$.

Positive and negative ToF-SIMS measurements for functionalized nanofibers were performed with a ToF-SIMS spectrometer from Charles Evans \& Associates. The sample was bombarded with pulsed $\mathrm{Ga}^{+}$ions $(15 \mathrm{keV})$. The secondary ions were accelerated to $\pm 3 \mathrm{keV}$ by applying a bias on the sample. The spreading of initial energies of the secondary ions is compensated by deflection in three electrostatic analyzers. A postacceleration of $7 \mathrm{keV}$ was applied at the detector entry. ${ }^{22}$ The analyzed area used in this work was a square of $120 \times$ $120 \mu \mathrm{m}$, and the data acquisition time was $5 \mathrm{~min}$. The powders were pressed on double-face silver tape. ToF-SIMS measurements for pristine or oxidized nanofibers were performed with an IONTOF V spectrometer. The sample was bombarded with pulsed $\mathrm{Bi}_{3}{ }^{+}$ions $(30 \mathrm{keV})$. The analyzed area used in this work was a square of $500 \times 500 \mu \mathrm{m}$ and the data acquisition time was $60 \mathrm{~s}$. On insulating samples (such as the pure clusters), charge effects were compensated by means of an interlaced pulsed electron flood gun $\left(E_{\mathrm{k}}=20 \mathrm{eV}\right)$. With these parameters, the primary ion dose density was lower than $10^{11} \mathrm{Bi}^{+}$per $\mathrm{cm}^{2}$. The powders were pressed with a spatula onto a double-sided silver adhesive sheet.

TGA analyses of the clusters placed into alumina containers were recorded on a SDT 2960 simultaneous DSC-TGA instrument from TA Instruments, with a heating ramp of $10{ }^{\circ} \mathrm{C} \mathrm{min}{ }^{-1}$ under $\mathrm{N}_{2}$ flow $\left(100 \mathrm{ml} \mathrm{min}^{-1}\right)$ to $900{ }^{\circ} \mathrm{C}$.

SEM images were obtained with a DSM982 Gemini FEG Microscope from LEO, equipped with a Phoenix CDU LEAP EDXS detector. The samples were fixed using double-sided conducting adhesive tape onto $5 \mathrm{~mm}$ diameter Al stubs from Agar Scientific.

TEM images were obtained with a LEO 922 OMEGA energy filter microscope. A drop of the sample suspended in hexane 
was deposited on a copper grid-supported holey carbon film, and dried under vacuum at room temperature before analysis.

\subsection{Magnetic characterization}

Magnetization measurements were carried out with the Vibrating Sample Magnetometry option of a Cryogenic Ltd closed cycle measurement system. Hysteresis loops in the range of $\pm 12 \mathrm{~T}$ of the functionalized MWCNT and CNF powders with deposited alloy nanoparticles with $\left[\mathrm{HFeCo}_{3}(\mathrm{CO})_{12}\right]$ as a precursor were measured at 5 and $300 \mathrm{~K}$ for all samples. The magnetic moment was measured as a function of temperature between 5 and $300 \mathrm{~K}$ upon heating, both after cooling in zero field (ZFC) and cooling in a $0.01 \mathrm{~T}$ per $\mu_{0}$ field (FC).

\section{Results and discussion}

\subsection{Surface functionalization of CNF and MWCNT}

Two different carbon materials (CNF and MWCNT) were functionalized on their surfaces for the grafting of metal clusters. These were selected for their availability (compared to SWCNT or isolated graphene sheets) and different reactivity (CNF exposing edges of graphene planes at the surface, while MWCNT being made of concentric layers). Two different strategies were envisaged as illustrated in Fig. 1. In the first case, chelating phosphine groups were introduced in three steps according to our previous work. ${ }^{7}$ In the second strategy, positively charged ammonium groups were formed. In both cases, carboxylic acid groups conversion into acyl chlorides followed by formation of amide bonds through reactions with diamines are the common steps. The available terminal amine group can then be converted into a bidentate phosphine by the combined action of formaldehyde and diphenylphosphine in the first case, or an ammonium group by quaternization in the second case. Proof of the covalent nature of these strategies was obtained previously by model studies in solution and solid-state NMR characterization. ${ }^{7,25}$ Due to the use of a diamine, it is possible to have a monodentate or bidentate surface attachment via amidation..$^{23,24}$ The loop structure is only possible for a high density of acyl chloride groups. Its only drawback is the loss of this function for further functionalization, i.e. lowering the final grafting yield. Here the samples were characterized by elemental analysis, XPS and $\mathrm{N}_{2}$ physisorption, as described below. Thermogravimetric Analyses (TGA) could not be used to determine grafting yield as each step is not $100 \%$ selective and because of the occurrence of loop structures. Similarly, vibrational spectroscopies (infrared, Raman) did not show the desired functions as they were lost in the absorption background.

3.1.1 Characterization of oxidized CNF and MWCNT supports. XPS analyses showed that the pristine CNF-LHT and CNF-PS present an $\mathrm{O} / \mathrm{C}$ ratio equal to 0.003 and 0.025 , respectively, while CNF-OX presents an $\mathrm{O} / \mathrm{C}$ ratio equal to 0.116 . In the case of MWCNT, preliminary oxidation studies, with nitric acid alone or with a mixture of sulfuric and nitric acids, were realized on small quantities of Nanocyl MWCNT. ${ }^{26}$ Here, MWCNTs were oxidized with concentrated nitric acid under reflux for variable durations (see ESI $\dagger$ ). The increase in the number of acid functions was followed by XPS, via quantification of O1s and C1s peaks. The starting MWCNTs (unreacted) have an $\mathrm{O} / \mathrm{C}$ surface ratio equal to 0.006 . The highest value $(\mathrm{O} / \mathrm{C}$ surface ratio $=$ 0.085) was obtained for 24 hours of acid treatment. For the other reaction times, $\mathrm{O} / \mathrm{C}$ ratios were very similar and in the range $0.055-0.060$, i.e. higher than those of pristine MWCNT. These numbers are lower than those with CNF-OX, as expected due to less exposed graphene edges. The selected treatment duration for the samples undergoing further steps was fixed for $2 \mathrm{~h}$ because MWCNT were partially damaged or destroyed with longer treatments (see $\mathrm{ESI} \dagger$ ).

Elemental analysis of the oxidized supports showed an expected increase in the percentage of oxygen (Table 1). In this case, oxygen was determined by direct measurement, not by difference. If we compare the O/C ratios determined by XPS and by elemental analysis for the oxidized samples, similar values were obtained for MWCNT-OX but in the case of CNF-OX, the ratio determined by elemental analysis was much lower than that determined by XPS. This result is in line with the fact that,

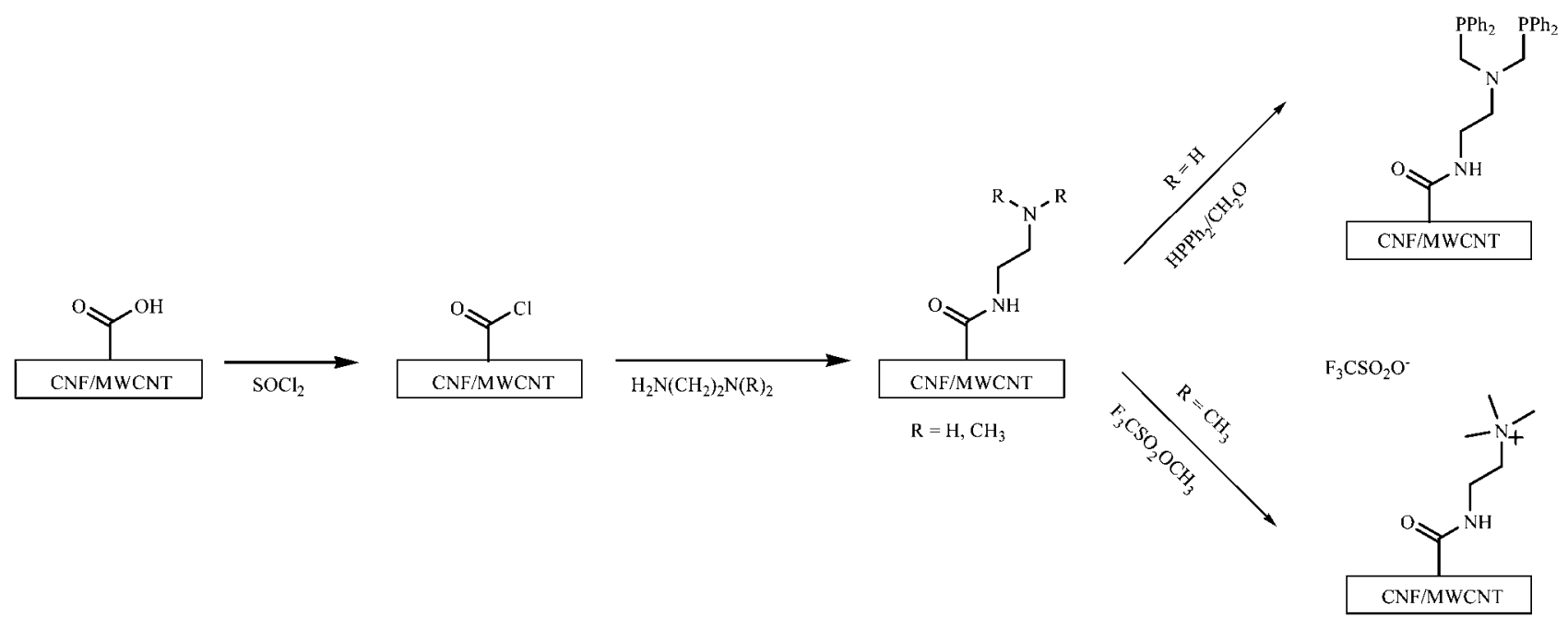

Fig. 1 Functionalization of CNF and MWCNT. 
Table 1 Elemental analyses and molar $\mathrm{O} / \mathrm{C}$ ratios for carbon supports before and after oxidation

\begin{tabular}{llllllll}
\hline Support & $\mathrm{C}(\mathrm{wt} \%)$ & $\mathrm{H}(\mathrm{wt} \%)$ & $\mathrm{N}(\mathrm{wt} \%)$ & $\mathrm{O}(\mathrm{wt} \%)$ & $\mathrm{O} / \mathrm{C}(\mathrm{mass})$ & $\mathrm{O} / \mathrm{C}(\mathrm{mol})$ & $\mathrm{XPS} \mathrm{O} / \mathrm{C}(\mathrm{mol})$ \\
\hline CNF-PS & 95.76 & 1.44 & 0.88 & 0.36 & 0.004 & 0.003 & 0.025 \\
CNF-OX & 93.43 & 1.32 & 0.91 & 1.75 & 0.019 & 0.014 \\
MWCNT & 87.68 & 1.21 & $<0.10$ & 0.28 & 0.003 & 0.002 \\
MWCNT-OX & 88.61 & 0.66 & 0.23 & 4.81 & 0.054 & 0.041 & 0.006 \\
\hline
\end{tabular}

because the radius of the MWCNT is in the range of a few nanometers, XPS behaves in this case as a bulk analysis.

3.1.2 Characterization of CNF and MWCNT functionalized with $-\mathbf{P P h}_{2}$ and $-\mathbf{N M e}_{3}{ }^{+}$. After each functionalization step, a black powder was obtained and characterized by XPS (Tables 2 and 3). The reaction yield was never $100 \%$ for each step, starting from the reaction with $\mathrm{SOCl}_{2}$, which only occurs on carboxylic acid groups. After each step, the heteroatom of interest was observed on the surface. Again, the numbers are higher on CNF than those on MWCNT. In the ammonium functionalized samples, the $\mathrm{F} / \mathrm{N}$ ratio must be equal to 1.5 and the $\mathrm{S} / \mathrm{N}$ ratio equal to 0.5 if the counter-ion $\left(\mathrm{CF}_{3} \mathrm{SO}_{3}{ }^{-}\right)$is present in proportional quantity with respect to the ammonium groups. This is indeed roughly the case.

Elemental analysis of CNF- $\mathrm{PPh}_{2} / \mathrm{NMe}_{3}{ }^{+}$and $\mathrm{MWCNT}-\mathrm{PPh}_{2} /$ $\mathrm{NMe}_{3}{ }^{+}$is summarized in Table 4 . If we compare the $\mathrm{P} / \mathrm{C}$ ratios determined by XPS and by elemental analysis for the $-\mathrm{PPh}_{2}$ functionalized samples, similar values were obtained for MWCNT-PPh ${ }_{2}$ but in the case of CNF- $\mathrm{PPh}_{2}$, the ratio determined by elemental analysis was much lower than that determined by XPS. This stems from considerations discussed above, related to the radius of MWCNT in the nanometre range making XPS a bulk analysis.

Nitrogen adsorption isotherms were recorded for representative samples at each step of the functionalization (Fig. 2(a) and 3(a)). The specific surface area was found to decrease on going from the pristine CNF to the phosphine or ammoniumfunctionalized ones. The surface area calculated using the BET equation is given in Table 5. By going from the CNF-PS to the CNF-OX, the specific surface area decreased significantly. The next step (CNF-Cl sample) displayed a slight decrease. In the subsequent steps no further decrease in specific surface area was observed. In the end, a nearly mesoporous-only material was obtained (Fig. 2(b)). In the case of MWCNT, the major

Table 2 XPS results for CNF functionalization

XPS

atomic

ratio CNF-OX CNF-Cl CNF-NH ${ }_{2}$ CNF-NMe ${ }_{2}$ CNF-PPh $_{2}$ CNF-NMe $_{3}{ }^{+}$

\begin{tabular}{lllllll}
\hline $\mathrm{O} / \mathrm{C}$ & 0.116 & 0.118 & 0.102 & 0.090 & 0.078 & 0.099 \\
$\mathrm{Cl} / \mathrm{C}$ & 0.007 & 0.019 & 0.014 & 0.010 & 0.004 & 0.003 \\
$\mathrm{~N} / \mathrm{C}$ & 0.008 & 0.030 & 0.073 & 0.058 & 0.035 & 0.034 \\
$\mathrm{P} / \mathrm{C}$ & - & - & - & - & 0.010 & - \\
$\mathrm{S} / \mathrm{C}$ & - & 0.006 & 0.005 & 0.004 & 0.001 & 0.010 \\
$\mathrm{~F} / \mathrm{C}$ & - & - & - & - & - & 0.048 \\
$\mathrm{~F} / \mathrm{N}$ & - & - & - & - & - & 1.407 \\
$\mathrm{~S} / \mathrm{N}$ & - & - & - & - & - & 0.310
\end{tabular}

difference was also in the oxidation step. In this case, the specific surface area increased. This increase in specific surface area can be ascribed to three effects: fragmentation, surface roughening and/or opening of the inner tubes. ${ }^{27,28}$ Using TEM, surface roughening was observed to be the major effect in our samples. Open ended tubes were present in small quantities and no fragmentation was observed. The subsequent steps $\left(\mathrm{MWCNT}-\mathrm{PPh}_{2}\right.$ or MWCNT-NMe ${ }_{3}^{+}$) displayed a further decrease of specific surface area. In the end, a totally mesoporous material was also obtained (Fig. 3(b)).

Knowing the specific surface area of the CNF-PPh ${ }_{2}\left(S_{\text {BET }}=\right.$ $32 \mathrm{~m}^{2} \mathrm{~g}^{-1}$ ) and the $\mathrm{P}$ wt\% determined by elemental analysis $(0.50 \mathrm{wt} \%)$, the number of $\mathrm{P}$ atoms present on the surface per unit of area can be calculated and corresponds to $\sim 3 \mathrm{P}$ per $\mathrm{nm}^{2}$. This value must be divided by 2 because the ligand is a bidentate phosphine (2P); so 1.5 anchoring sites per $\mathrm{nm}^{2}$ were present on the support. The same calculations have been made with MWCNT-PPh $2\left(S_{\text {BET }}=241 \mathrm{~m}^{2} \mathrm{~g}^{-1}\right.$ and $\mathrm{P}$ wt $\left.\%=1.11\right)$, and 0.45 anchoring sites per $\mathrm{nm}^{2}$ were found. In the case of ammoniumfunctionalized support, the same calculations from $\mathrm{N}$ wt\% do not seem reliable because nitrogen is already present on the pristine CNF or MWCNT. It is difficult to compare these results with data from the literature because only one group also quantified amounts of P-groups on nanotubes, but these were not covalently linked: Suri et al. ${ }^{\mathbf{1 1}}$ found 0.9 tertiary phosphines per 100 carbon atoms for SWCNT-PPh ${ }_{3}$ mixtures.

3.1.3 XPS in pseudo in situ mode: species evolution under thermal treatment. To determine the different types of functions present on the surface of the supports, it is possible to decompose XPS peaks into several components. However, it has to be noted that small differences between the binding energies of atoms associated with the different functions make the decomposition of peaks very delicate, particularly for $\mathrm{C} 1 \mathrm{~s}$ and $\mathrm{O}$ 1s components. ${ }^{5}$ In addition, the evolution of surface species during thermal treatment was studied by XPS in pseudo in situ mode and compared with the results obtained after heating under the same conditions in a tubular oven. This study was carried out with $\mathrm{CNF}$, and the results can be reasonably extrapolated to MWCNT.

3.1.3.1 Ammonium functionalization. The C 1 s peak can be fitted with a number of components, but due to the strength of the shake-up tail relative to the low concentration of different oxide or amide groups, it is not very reliable. After heating the $\mathrm{NMe}_{3}{ }^{+}$sample to $300{ }^{\circ} \mathrm{C}$ (Fig. 4(a)), a decrease at 292-293 eV which corresponds to $\mathrm{CF}_{3}$ and another decrease at 286-289 eV which corresponds to oxygen atoms bound to carbon were observed. These results show that oxygenated functions are destroyed during thermal treatment. The $O 1$ s spectrum is 
Table 3 XPS results for MWCNT functionalization

\begin{tabular}{|c|c|c|c|c|c|c|c|}
\hline $\begin{array}{l}\text { XPS atomic } \\
\text { ratio }\end{array}$ & MWCNT & MWCNT-OX & MWCNT-Cl & MWCNT-NH ${ }_{2}$ & MWCNT-NMe ${ }_{2}$ & MWCNT-PPh 2 & MWCNT-NMe ${ }_{3}^{+}$ \\
\hline $\mathrm{O} / \mathrm{C}$ & 0.006 & 0.055 & 0.045 & 0.037 & 0.041 & 0.038 & 0.054 \\
\hline $\mathrm{N} / \mathrm{C}$ & - & 0.005 & 0.006 & 0.018 & 0.017 & 0.013 & 0.013 \\
\hline $\mathrm{P} / \mathrm{C}$ & - & - & - & - & - & 0.004 & - \\
\hline $\mathrm{S} / \mathrm{C}$ & - & - & - & - & - & - & 0.004 \\
\hline $\mathrm{S} / \mathrm{N}$ & - & - & - & - & - & - & 0.323 \\
\hline
\end{tabular}

Table 4 Elemental analysis of $-\mathrm{PPh}_{2}$ and $-\mathrm{NMe}_{3}{ }^{+}$functionalized supports

\begin{tabular}{|c|c|c|c|c|c|c|c|c|c|}
\hline Support & $\mathrm{C}(\mathrm{wt} \%)$ & $\mathrm{H}(\mathrm{wt} \%)$ & $\mathrm{N}(\mathrm{wt} \%)$ & $\mathrm{S}(\mathrm{wt} \%)$ & $\mathrm{Cl}(\mathrm{wt} \%)$ & $\mathrm{P}(\mathrm{wt} \%)$ & F (wt\%) & $\mathrm{P}$ or N/C (mass) & $\mathrm{P}$ or $\mathrm{N} / \mathrm{C}(\mathrm{mol})$ \\
\hline CNF-PPh 2 & 92.53 & 1.34 & 1.08 & 2.49 & 1.14 & 0.50 & - & 0.005 & 0.002 \\
\hline $\mathrm{CNF}^{-\mathrm{NMe}_{3}}{ }^{+}$ & 93.76 & 1.31 & 0.93 & 2.43 & 0.19 & - & 0.94 & 0.010 & 0.008 \\
\hline MWCNT-PPh 2 & 86.61 & 0.87 & 1.25 & $<0.10$ & 0.38 & 1.11 & - & 0.013 & 0.005 \\
\hline 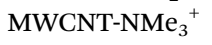 & 82.49 & 0.80 & 0.85 & 2.99 & 0.39 & - & 1.91 & 0.010 & 0.009 \\
\hline
\end{tabular}

decomposed into two main peaks (Fig. 4(b)): oxygen doubly bound to carbon $(\mathrm{C}=\mathrm{O})$ in quinones, ketones, and aldehydes at $531.5-532 \mathrm{eV}$, and oxygen singly bound to carbon (C-O) in ethers and phenols at about $533 \mathrm{eV}$. Because oxygen atoms in esters, carboxyls, anhydrides, and pyrones are involved in both single and double bonds with carbon atoms, these groups

(a)

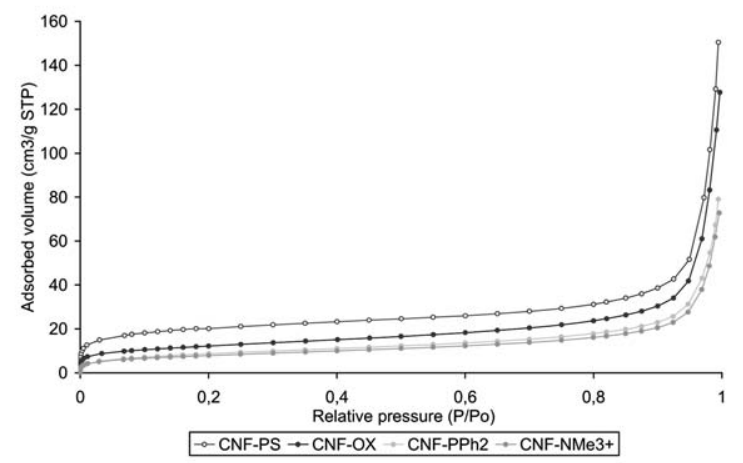

(b)

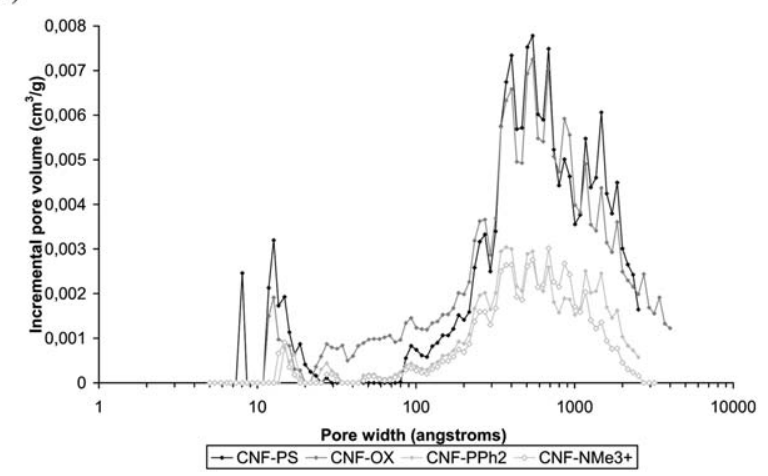

Fig. 2 (a) Nitrogen adsorption isotherms and (b) pore size distribution for functionalized CNF. contribute to both the above-mentioned peaks. ${ }^{29}$ Moreover, oxygen atoms bound to sulfur (SOx) are also present and contribute to the first component. The intensities of both single and double-bond peaks decreased after heating to $300{ }^{\circ} \mathrm{C}$, which can be attributed to the disappearance of carboxyl and SOx groups. A third peak was observed at $535-536 \mathrm{eV}$. In the

(a)

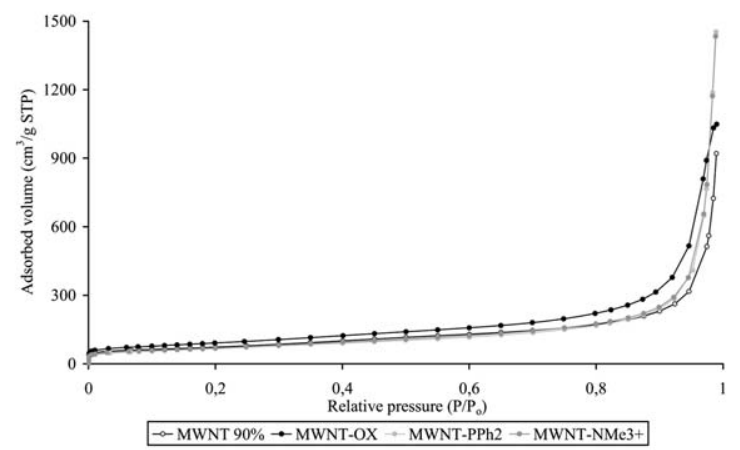

(b)

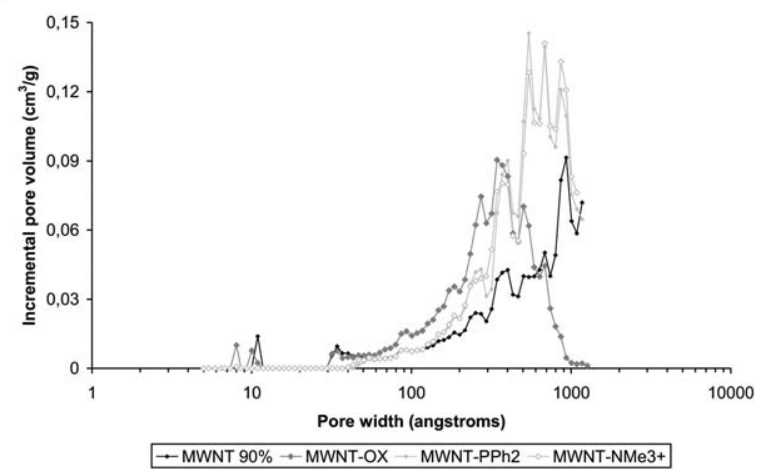

Fig. 3 (a) Nitrogen adsorption isotherms and (b) pore size distribution for functionalized MWCNT. 
Table 5 Textural properties of the different supports

\begin{tabular}{|c|c|c|c|}
\hline & $S_{\mathrm{BET}}\left(\mathrm{m}^{2} \mathrm{~g}^{-1}\right)$ & $V_{\text {pore }}\left(\mathrm{cm}^{3} \mathrm{~g}^{-1}\right)$ & $D_{\text {pore }}(\mathrm{nm})$ \\
\hline CNF-LHT & 35 & 0.18 & 26 \\
\hline CNF-PS & 72 & 0.21 & 29 \\
\hline CNF-OX & 43 & 0.19 & 26 \\
\hline CNF-Cl & 29 & 0.10 & 23 \\
\hline CNF-NH ${ }_{2}$ & 28 & 0.10 & 22 \\
\hline $\mathrm{CNF}-\mathrm{NMe}_{2}$ & 30 & 0.10 & 20 \\
\hline CNF-PPh ${ }_{2}$ & 32 & 0.11 & 20 \\
\hline${\mathrm{CNF}-\mathrm{NMe}_{3}}^{+}$ & 29 & 0.11 & 21 \\
\hline MWCNT & 257 & 1.42 & 22 \\
\hline MWCNT-OX & 325 & 1.62 & 20 \\
\hline MWCNT-PPh 2 & 241 & 2.26 & 36 \\
\hline MWCNT-NMe ${ }_{3}{ }^{+}$ & 249 & 2.23 & 33 \\
\hline
\end{tabular}

literature, ${ }^{6}$ this peak is often attributed to water, but in our case, this interpretation does not seem plausible because the sample was heated at $300{ }^{\circ} \mathrm{C}$ in the reaction chamber of the XPS instrument and directly introduced into the analytical chamber under ultra-high vacuum without any air contact. As shown in Fig. 4(c), decomposition of $\mathrm{N} 1 \mathrm{~s}$ peak results in two components at $399-400 \mathrm{eV}$ corresponding to amine or amide and one component at $402.7 \mathrm{eV}$ assigned to ammonium groups. After heating at $300^{\circ} \mathrm{C}$, all peaks decreased, and more particularly the ammonium peak; thus the functional group bearing the charge is destroyed. The decomposition of the $\mathrm{Cl} 2 \mathrm{p}$ peaks (Fig. 4(d)) reveals two non-equivalent chlorine sites. These two sites were present in the starting material but in much lower proportion. After addition of $\mathrm{SOCl}_{2}$, the concentration of the two sites increased. The attribution was difficult. According to the literature, ${ }^{30,31}$ the more intense $\mathrm{Cl} 2 \mathrm{p}$ component at $200.6 \mathrm{eV}$ is typical of chlorine participating in organic $\mathrm{C}-\mathrm{Cl}$ bonds. The less intense component with a lower binding energy of $198.8 \mathrm{eV}$ can be assigned to chloride ions which are ionically bonded to the surface. After heating, the two components decreased and the first at $198.8 \mathrm{eV}$ disappeared. As shown in Fig. 4(e), decomposition of the S 2p peak results in two components. The peak at
$164 \mathrm{eV}$ corresponds to sulfide and was present from the beginning. The second peak at $168.5 \mathrm{eV}$ can be attributed to the sulfur from triflate and also, in smaller proportion, to $\mathrm{SOCl}_{2}$ molecules, or other $\mathrm{S}$ containing species, including $\mathrm{SO}_{2}{ }^{30}$ After heating, the first peak was not affected whereas the second one at $168.5 \mathrm{eV}$ decreased strongly meaning that the counter-ion was lost. There was no significant difference between the results obtained in the tubular oven in the ex situ mode (see ESI $\dagger$ ) and those obtained in the reaction chamber of the Kratos instrument. To conclude, under thermal treatment the anchoring arms were affected but the different elements which made up these arms remained present on the surface as contaminants.

3.1.3.2 Phosphine functionalization. In the case of CNF-PPh the following observations can be made. Concerning the $\mathrm{C} 1 \mathrm{~s}$ peak after heating at $300{ }^{\circ} \mathrm{C}$ (Fig. 5(a)), a decrease was observed at $286-289 \mathrm{eV}$ which corresponds to oxygen atoms bound to carbon. The decomposition of the $\mathrm{O} 1 \mathrm{~s}$ spectrum results in three components (Fig. 5(b)) as for CNF-NMe ${ }_{3}^{+}$. The intensities of oxygen atoms singly and doubly bound to carbon peaks both decrease after heating to $300{ }^{\circ} \mathrm{C}$, which can be attributed to decomposition of carboxyl groups. As shown in Fig. 5(c), decomposition of the $\mathrm{N} 1 \mathrm{~s}$ peak results in two components, at 400.7 and $402.8 \mathrm{eV}$, corresponding to amine or amide and to ammonium, respectively. The latter was very small compared to the one above which makes sense. After heating at $300{ }^{\circ} \mathrm{C}$, the peak at $402.8 \mathrm{eV}$ disappeared and the peak at $400.7 \mathrm{eV}$ split into two components at 399.5 and $401.4 \mathrm{eV}$. The decomposition of the $\mathrm{Cl}$ 2p peak (Fig. 5(d)) reveals two inequivalent chlorine sites. The explanation is the same as for $\mathrm{CNF}-\mathrm{NMe}_{3}{ }^{+}$. Finally, as shown in Fig. 5(e), decomposition of the $\mathrm{P} 2 \mathrm{p}$ peak results in two components: one at $131 \mathrm{eV}$ which corresponds to diphenylphosphine and the second at $133 \mathrm{eV}$ which corresponds probably to oxidized phosphine. ${ }^{32}$ This oxidation is assumed to occur during the sample preparation for the XPS analysis, and not during the synthesis, because all steps were realized under inert atmosphere. After heating at $300{ }^{\circ} \mathrm{C}$, only the peak at $133 \mathrm{eV}$ remains. There was again no significant difference when comparing the results obtained in the tubular oven (see ESI $\dagger$ ) (a)

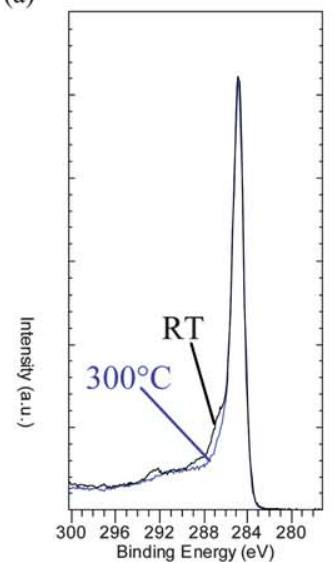

(b)

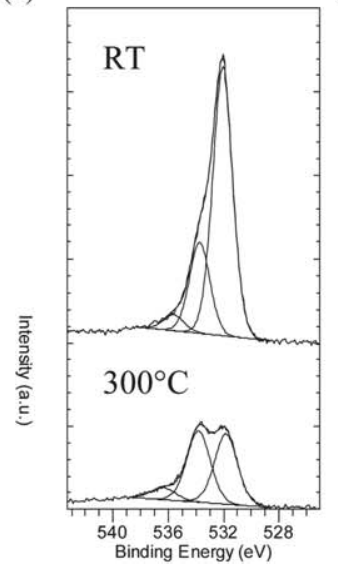

(c)

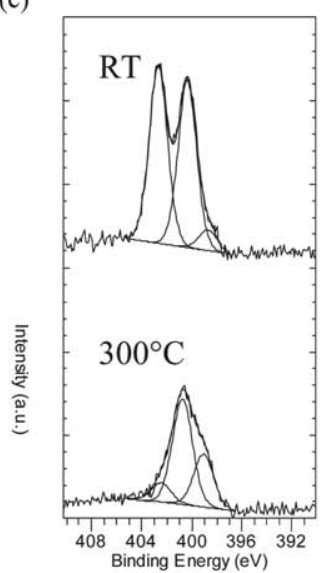

(d)

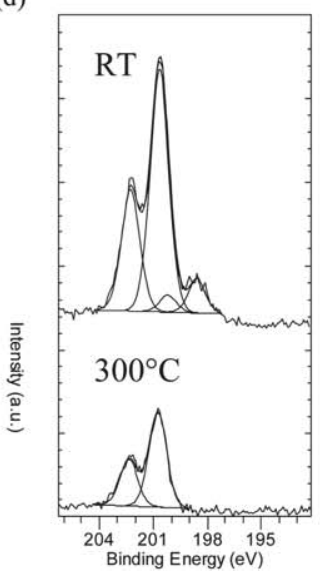

(e)

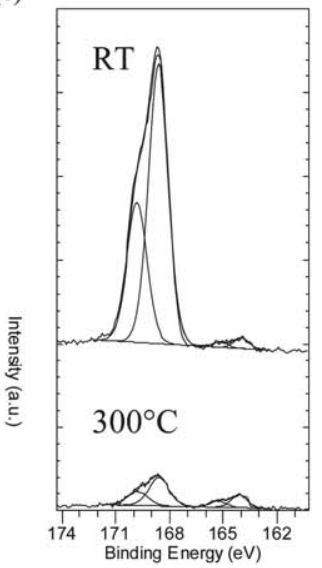

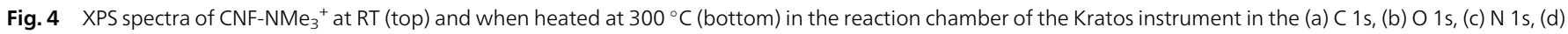
$\mathrm{Cl} 2 \mathrm{p}$, and (e) $\mathrm{S} 2 \mathrm{p}$ regions. 
(a)

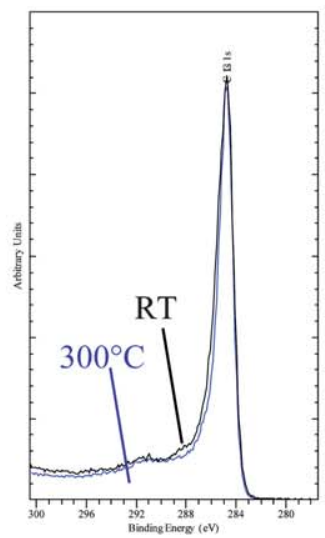

(b)

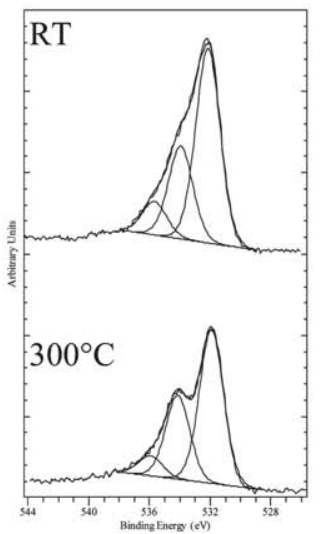

(c)

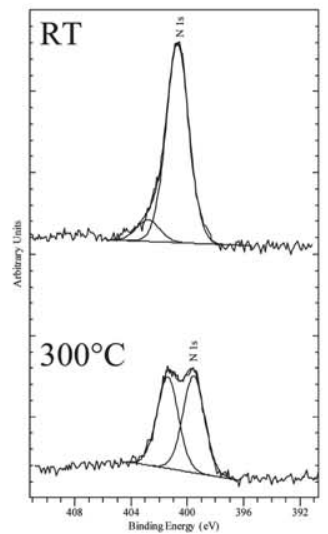

(d)

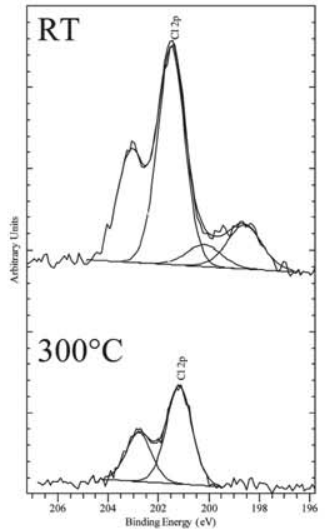

(e)

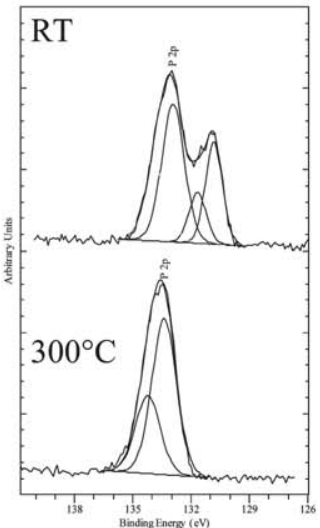

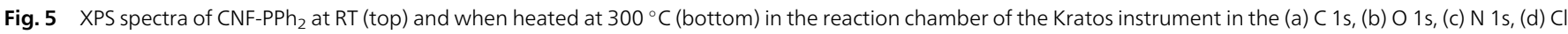
$2 p$, and (e) $P$ 2p regions.

with those in the reaction chamber of the Kratos instrument. So, the conclusion was the same as for ammonium functionalized CNF: thermal treatment partially destroyed the functionalization arm but some elements nevertheless remained present on the surface as contaminants.

\subsection{Deposition of bimetallic nanoparticles on CNF and MWCNT via decomposition of metal clusters}

Clusters $\left[\mathrm{HFeCo}_{3}(\mathrm{CO})_{12}\right](1)$ and $\left(\mathrm{NEt}_{4}\right)\left[\mathrm{FeCo}_{3}(\mathrm{CO})_{12}\right]$ (2) were prepared as described previously. ${ }^{20}$ The two clusters were incorporated on the functionalized or pristine supports by stirring in a mixture of toluene, THF and/or acetone. The loaded supports were recovered by filtration and washed, and any remaining (unreacted) clusters were found in the filtrates/ washings. Thus the maximum metal loading can be controlled by the initial amount of cluster dissolved in the impregnating solution. The metal loading was determined by Co atomic absorption analysis of the synthesis filtrates (Fig. S5 in ESI $\dagger$ ). The loading was directly affected by the solvent(s) used. Infrared spectroscopy confirmed, in the case of cluster $\mathbf{1}$, that it is protonated in toluene, deprotonated in $\mathrm{THF}$, and that an equilibrium between both forms is obtained in acetone (Table S6, see ESI $\dagger$ ). A higher incorporation yield has been thus obtained on $\mathrm{CNF}-\mathrm{PPh}_{2}$ in toluene and on $\mathrm{CNF}-\mathrm{NMe}_{3}{ }^{+}$in $\mathrm{THF}$ (see Fig. S5(a)†). We believe that the cluster gets covalently bound on the $\mathrm{CNF}-\mathrm{PPh}_{2}$ surface by $\mathrm{CO} / \mathrm{PPh}_{2}$ ligand exchange, but that it is immobilized by ion-pairing on the cationic surface of $\mathrm{CNF}^{-\mathrm{NMe}_{3}}{ }^{+}$in THF when $\mathbf{1}$ is deprotonated and hence negatively charged. The cluster-surface interaction is thus strong and specific. However, the incorporation results obtained in acetone were very low probably due to the high solubility of the cluster in this solvent. Armstrong et al. also observed that this cluster was better incorporated on inorganic supports from toluene than from acetone. ${ }^{33}$ For the oxidized or pristine supports, only toluene or THF was used to favour incorporation. For pristine CNF, all engaged clusters were incorporated except for CNF-LHT in THF. For the oxidized support, the incorporation yield $(\sim 70 \%)$ was the same with both solvents.

In the case of cluster 2 , which is negatively charged, the incorporation yield was small in every case but, here, the best results were obtained when a mixture of acetone-THF was used. There was no incorporation on CNF-LHT and CNF-PS for cluster 2. This result is consistent with the fact that the pristine nanofibers have no or very few surface functions. With oxidized nanofibers, the incorporation yield was approximately the same as on ammonium-functionalized CNF. When comparing clus-

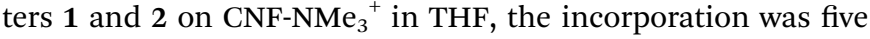
times higher for cluster 1. Both clusters were deprotonated but cluster 2 had a counter-ion which stabilized the cluster in solution. This counter-ion probably competes with the support, which explains the low yield of incorporation. Cluster 2 also was incorporated on CNF-PPh $\mathrm{P}_{2}$ but to a lesser extent than on CNF$\mathrm{NMe}_{3}{ }^{+}$. In the case of the charged support, the interaction with the negatively charged cluster 2 must be electrostatic, while for $\mathrm{CNF}-\mathrm{PPh}_{2}$ a ligand exchange mechanism must occur: some carbonyl ligands are lost against the surface phosphine groups. Counter-ions must remain in the vicinity as a charge balancing group.

For MWCNT, only THF or toluene and mixture of THFacetone were selected to perform incorporation of cluster 1 and cluster 2 respectively. When comparing results for cluster 1 on phosphine and ammonium functionalized CNF with MWCNT, the incorporation yield was the same. In the case of oxidized MWCNT, the incorporation yield on MWCNT-OX was higher than that on CNF-OX. This is due to a higher oxygen content in MWCNT ( $c f$. elemental analyses). For pristine nanotubes, the highest value was observed in THF. In the case of cluster 2, the incorporation yield was again lower than that for cluster $\mathbf{1}$. The values were the same for oxidized or phosphine functionalized supports if we compared nanotubes and nanofibers. This time, incorporation on pristine nanotubes occurred, which was not the case with pristine nanofibers. The incorporation yield for MWCNT-NMe ${ }_{3}{ }^{+}$was almost two times higher than that for $\mathrm{CNF}_{-\mathrm{NMe}}{ }_{3}^{+}$even if less functions were present at the surface in 
the former case. To conclude, we believe that on a CNF-PPh support, grafting occurs via ligand exchange while on CNF$\mathrm{NMe}_{3}{ }^{+}$, ion pairs are formed. When oxygenated functions are present, some chemical interactions can take place while on pristine supports only non-specific adsorption can occur.

Some solid samples were characterized by SIMS after cluster incorporation. Representative examples of obtained SIMS spectra are displayed in Fig. 6. The spectrum of the pure unsupported cluster 1 (Fig. 6(a)) consisted of several peaks, corresponding to the loss of ligands, which is a typical fragmentation pattern of such clusters. The highest mass peak was observed at $\mathrm{m} / \mathrm{z} 569$ and could be attributed to the fragment $\left[\mathrm{FeCo}_{3}(\mathrm{CO})_{12}\right]^{-}$and was followed by a series of peaks corresponding to $\mathrm{CO}$ losses up to $\mathrm{m} / \mathrm{z} 317\left(\left[\mathrm{FeCo}_{3}(\mathrm{CO})_{3}\right]^{-}\right)$. The spectrum of the same cluster incorporated on $\mathrm{CNF}-\mathrm{PPh}_{2}$ (Fig. 6(b)) displayed similar patterns as spectrum (a). It indicated that the cluster was molecularly intact at the surface of the support before activation. However, irrespective of the cluster, the functionalized support $\left(-\mathrm{PPh}_{2}\right.$ or $\left.-\mathrm{NMe}_{3}{ }^{+}\right)$or the solvent used, the same result was obtained. In contrast, in the case of oxidized or pristine supports, in spite of the fact that the two metals were present in the spectra, the typical cluster fragmentation patterns no longer exist, indicating cluster degradation during incorporation. This underlines the advantage of using functionalized supports to maintain the cluster intact at the surface.

(a)

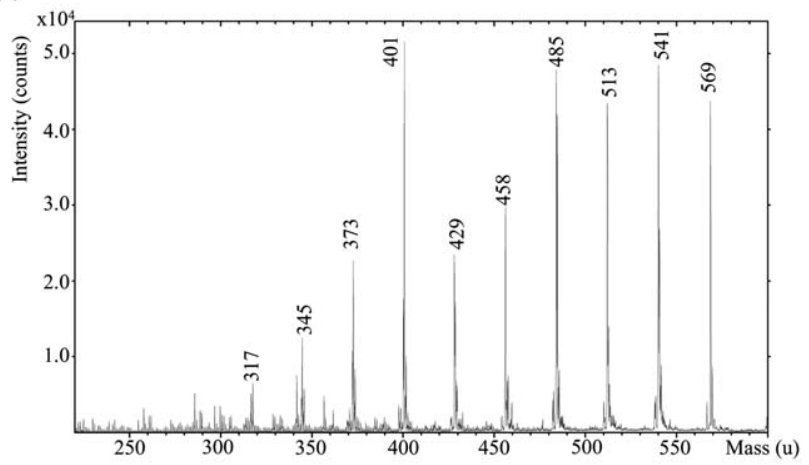

(b)

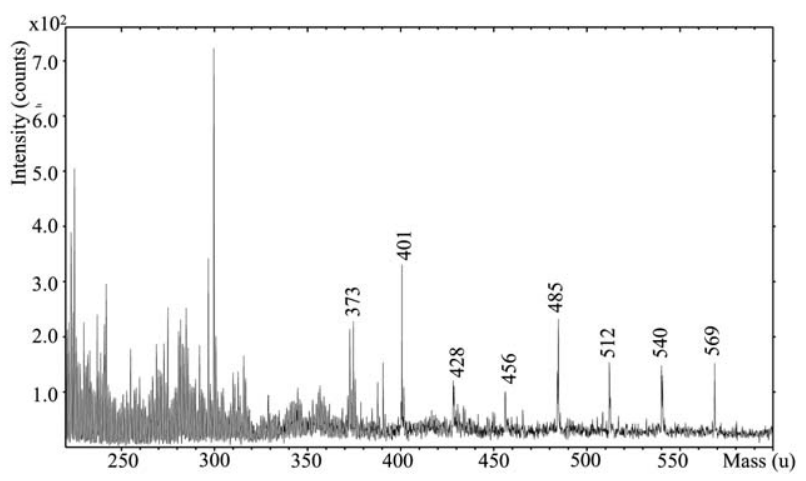

Fig. 6 Negative SIMS spectra of cluster $\left[\mathrm{HFeCO}_{3}(\mathrm{CO})_{12}\right]$ (1) before thermal treatment: (a) pure unsupported cluster as reference and (b) cluster $\mathbf{1}$ incorporated on CNF-PPh 2 .
After incorporation, thermal activation was performed at $200{ }^{\circ} \mathrm{C}$ for cluster 1 and $300{ }^{\circ} \mathrm{C}$ for cluster 2 , for one hour under $\mathrm{N}_{2}$, to convert metal clusters into supported metal particles. These temperatures correspond to the removal of ligands (see Table S7 in ESI $\dagger$ ). During thermal treatment, clusters are thus denuded of their CO ligands and metal atoms coalesce into nanoparticles. The interaction with the surface is not molecular anymore but some remaining fragment from the functionalization arms (which do not disappear completely as revealed by pseudo in situ XPS) might still play a stabilizing role.

The different samples were characterized by XPS before and after activation to determine the $\mathrm{Co} / \mathrm{C}$ surface ratios. The $\mathrm{Fe} / \mathrm{C}$ ratios could not be determined because the cobalt Auger line $\mathrm{L}_{3} \mathrm{M}_{45} \mathrm{M}_{45}$ and the Fe 2p XPS peak overlap each other, while the other Fe XPS peaks are not sensitive enough to be used. The XPS results are presented in Tables S8-S11 in ESI. $\dagger$ For both clusters, there is no or only small differences before and after activation which means that there was no or little agglomeration during thermal treatment. In the case of cluster 1 on CNF, the experimental $\mathrm{Co} / \mathrm{C}$ values were always higher than the calculated values. This result is consistent with the fact that only the surface is analyzed (it does not take into account all the carbon) in opposition to the calculated value which is a bulk value (all carbon being considered). When comparing CNF1, CNF3 and CNF12 (or CNF2 with CNF8, and CNF4 with CNF13), which correspond to the same metal loading and where only the support is different, it appears that the $\mathrm{Co} / \mathrm{C}$ ratio is significantly higher on functionalized than on pristine CNF, meaning that the cluster was better dispersed on a functionalized support. If we take into account the charge of cluster 1 in the different solvents, the highest $\mathrm{Co} / \mathrm{C}$ values are observed in toluene, where cluster 1 is electrically neutral, with $\mathrm{CNF}-\mathrm{PPh}_{2}$ which is also neutral (CNF12). An intermediate value was obtained when the cluster is charged (CNF13 and CNF14). Finally, in the presence of acetone (CNF15 and CNF16), the values were low due to low cluster incorporation from this solvent. The ratios between the experimental and the calculated ratios were also calculated to allow comparison between different samples with different metal loading, leading to the same conclusions.

In the case of cluster 1 on MWCNT, the experimental values were not or only slightly higher than the calculated values, which is also consistent with the fact that, in this case, XPS analysis behaves like a bulk method. For MWCNT, a better dispersion is expected on oxidized carbon nanotubes in toluene because its presents the highest Co/C values (MWCNT3).

In the case of cluster 2 on CNF, no metal was observed on non-functionalized carbon nanofibers (CNF17 and 18) as expected from the incorporation yield equal to zero. With the other supports, the same trends as for cluster $\mathbf{1}$ were observed. The highest $\mathrm{Co} / \mathrm{C}$ ratios were observed on oxidized or ammonium functionalized carbons, because this negatively charged cluster was better stabilized on charged supports.

Finally, the samples were characterized by TEM to determine the particle sizes after activation and to observe their dispersion. For all samples on carbon nanofibers, histograms of particle size distributions were realized (see ESI $\dagger$ ). First of all, 
when cluster 1 was introduced on pristine nanofibers, the particle sizes are not homogeneous (Fig. 7(a) and (b)), and the particles agglomerate seriously. Particles of predominantly 1$2 \mathrm{~nm}$ are seen but next to these, particles with a size larger than $500 \mathrm{~nm}$ are also present. The presence of Co and Fe in these big particles was confirmed by EDXS (ESI $\dagger$ ). Second, in the case of the same cluster incorporated on oxidized carbon nanofibers, small particles with a size between 1 and $11 \mathrm{~nm}$ are observed (Fig. 7(c)), with a majority of 1-2 nm particles. In Fig. 7(d) and (e) are shown the cases of the same cluster incorporated on functionalized nanofibers: small particles with a size less than 3 $\mathrm{nm}$ were obtained. These results proved the necessity of the support functionalization to prevent particle agglomeration and to obtain a surface with small particles homogeneously distributed. If we compare Fig. 7(e) and (f) where the difference is the solvent used and hence, the metal loading, particle sizes are higher when the metal loading is higher. We move from a size below $1 \mathrm{~nm}$ to a size of $1-11 \mathrm{~nm}$. In the case of cluster 2 on CNF-OX, very small particles $(<2 \mathrm{~nm})$ were present (Fig. 8(a)). When the same cluster was incorporated on CNF-NMe ${ }_{3}{ }^{+}$, the size distribution was approximatively the same (up to $3 \mathrm{~nm}$ ) (Fig. 8(b)). For both clusters on nanotubes, TEM did not show unambiguously the presence of particles, especially for functionalized supports, though XPS and cobalt atomic absorption had confirmed the presence of metals. For cluster 1, the size of particles decreased in the following order: MWCNT $>$ MWCNT-

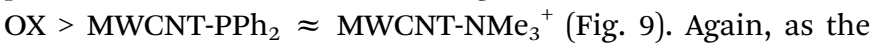
particle sizes are smaller on the functionalized supports, it shows the benefit of the anchoring point in avoiding agglomeration of clusters into bigger particles. For the sample on (a) $\mathrm{CNF} 2$

(c) CNF6

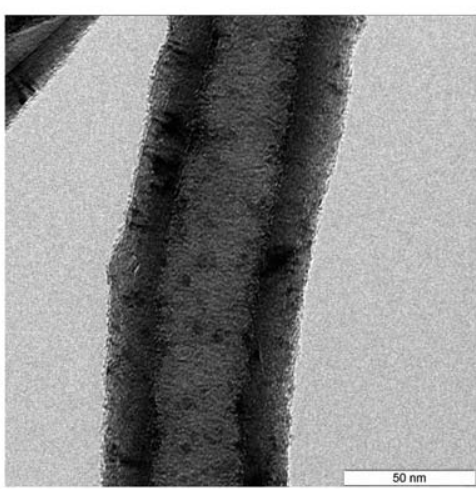

(e) CNF13
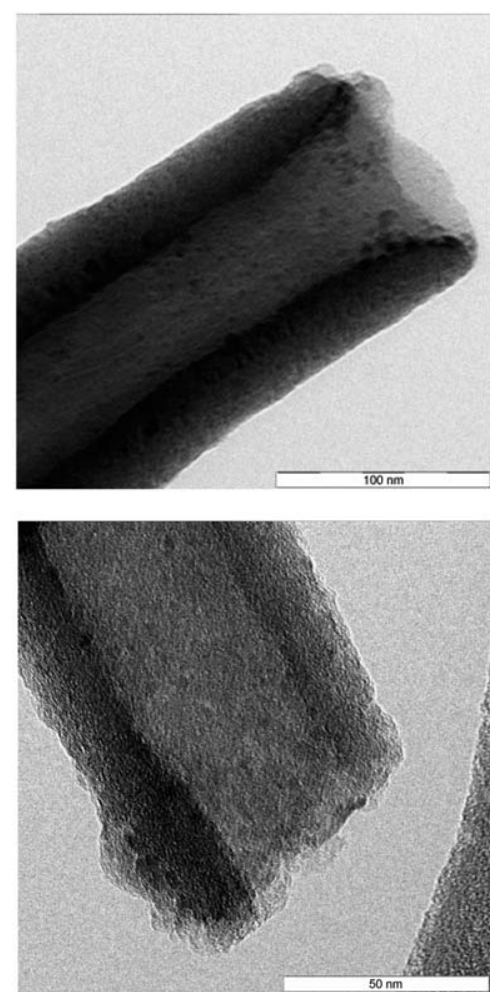

(b) $\mathrm{CNF} 2$

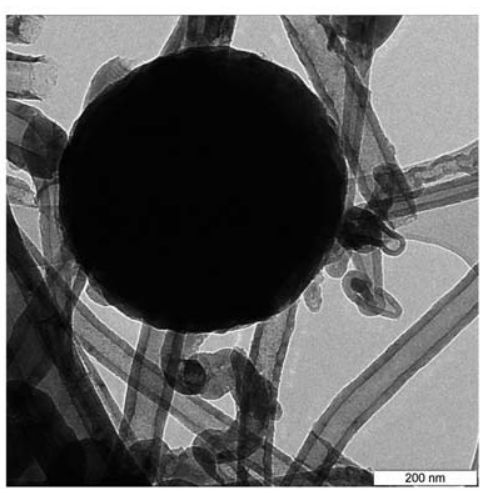

(d) CNF8

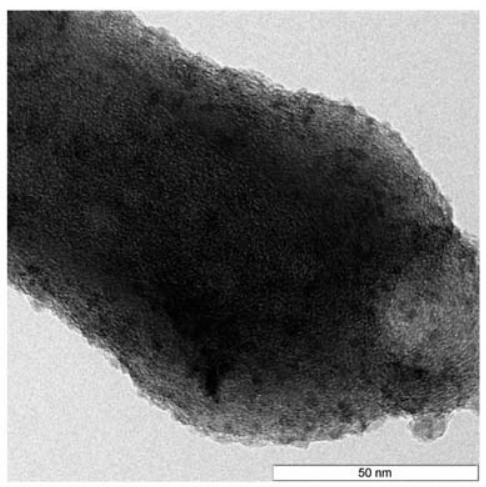

(f) $\mathrm{CNF} 12$

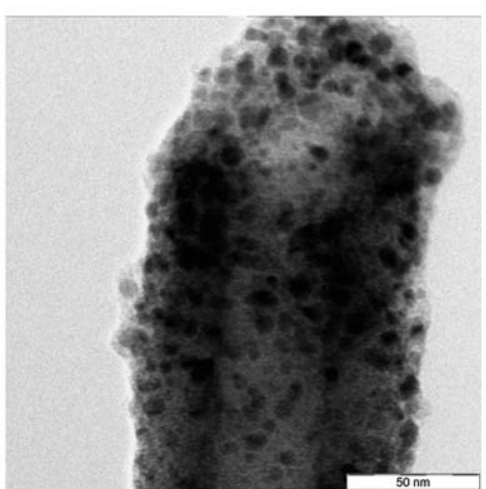

Fig. 7 TEM images of cluster $\left[\mathrm{HFeCO}_{3}(\mathrm{CO})_{12}\right](\mathbf{1})$ after thermal treatment: (a and b) on CNF-LHT, (c) on CNF-OX, (d) on CNF-NMe ${ }_{3}{ }^{+}$, (e) on CNF-PPh 2 in THF and (f) on CNF-PPh 2 in toluene. Metal loading: (a) 5 (b) 5 (c) 4 (d) 5 (e) 2.5 and (f) 5 wt\%. 
(a) CNF19

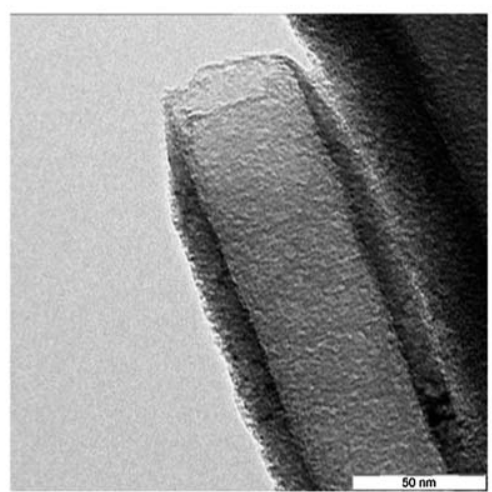

(b) CNF22

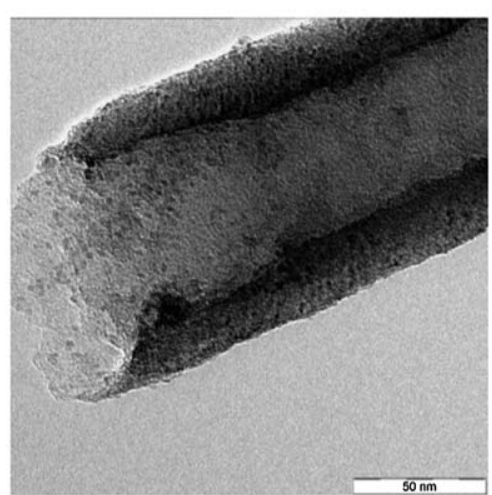

Fig. 8 TEM images of cluster $\left(\mathrm{NEt}_{4}\right)\left[\mathrm{FeCO}_{3}(\mathrm{CO})_{12}\right](\mathbf{2})$ after thermal treatment: (a) on CNF-OX and (b) on CNF-NMe ${ }_{3}^{+}$. Metal loading: (a) 1.6 and (b) 1.6 wt\%.

(a) MWCNT2

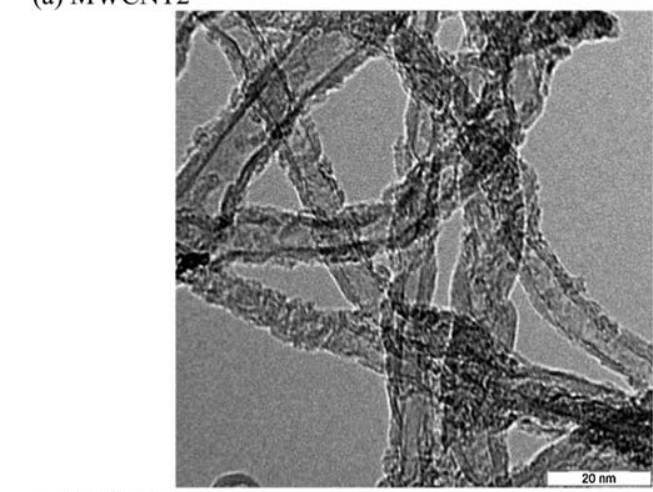

(c) MWCNT5

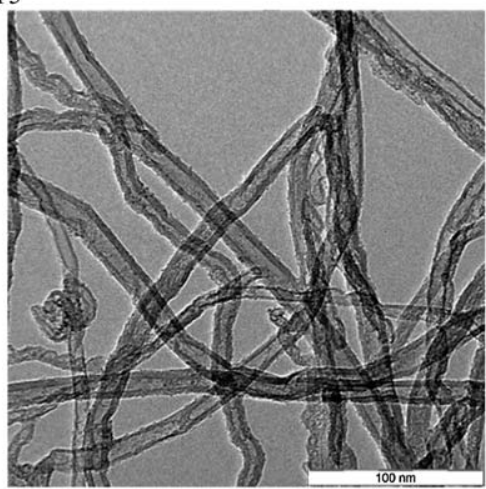

(b) MWCNT3

(d) MWCNT6
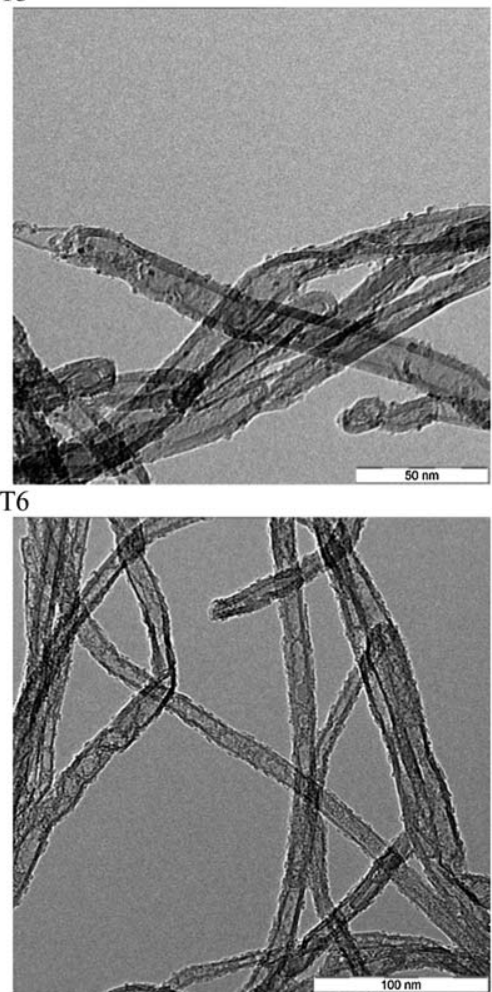

Fig. 9 TEM images of cluster $\left[\mathrm{HFeCO}_{3}(\mathrm{CO})_{12}\right]$ (1) after thermal treatment: (a) on MWCNT, (b) on MWCNT-OX, (c) on MWCNT-NMe ${ }_{3}{ }^{+}$and (d) on MWCNT-PPh 2 . Metal loading: (a) 4.6 (b) 5 (c) 5 and (d) 5 wt\%.

MWCNT-PPh ${ }_{2}$, the presence of cobalt was also proven by EDXS. In the case of cluster 2 on MWCNT (Fig. 10), no particle was visible even if cluster incorporation and thermal treatment took place successfully as described above, meaning that they had reached sub-nanometric sizes. No separated metallic entities were observed by microscopy. We believe that a templating effect of the fiber/tube diameter on the nanoparticle sizes exists, as unravelled previously in a slightly different context. ${ }^{26}$ Apart from that, a parallelism exists between the two types of carbonaceous materials, allowing the same methodology to be implemented to obtain carbon-supported bimetallic nanoparticles of very small sizes.

\subsection{Magnetic measurements}

Magnetic properties were determined for deposited nanoparticles derived from the $\left[\mathrm{HFeCo}_{3}(\mathrm{CO})_{12}\right]$ cluster on MWCNT and $\mathrm{CNF}$ functionalized with $\mathrm{PPh}_{2}$ and $\mathrm{NMe}_{3}{ }^{+}$, respectively. All four samples exhibit a ferromagnetic hysteresis already at room temperature, which is depicted in Fig. 11(a) and (b). Consequently, and in line with Fig. 11(e) which represents the zerofield cooled and field cooled susceptibility, exemplarily for MWCNT-PPh ${ }_{2}$, the blocking temperature is above room temperature. At room temperature, the saturation magnetization and the coercive field are mainly determined by the nature 
(a) MWCNT8

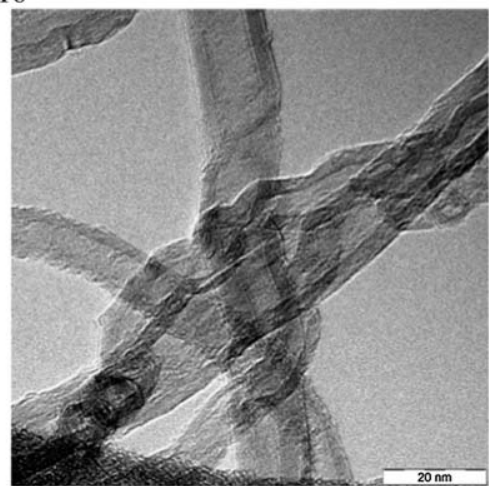

(b) MWCNT9

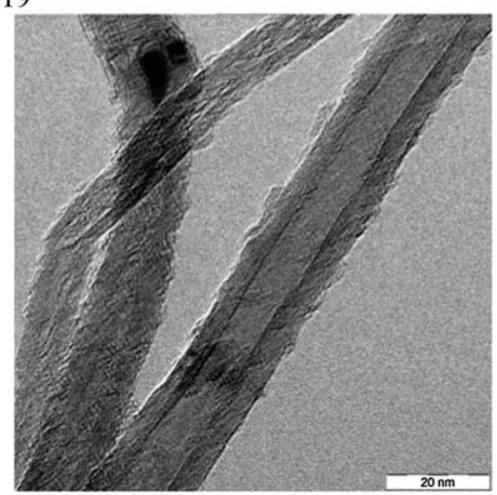

Fig. 10 TEM images of cluster $\left(\mathrm{NEt}_{4}\right)\left[\mathrm{FeCO}_{3}(\mathrm{CO})_{12}\right]$ (2) after thermal treatment: (a) on MWCNT-OX and (b) on MWCNT-NMe ${ }_{3}^{+}$. Metal loading: (a) 1.8 and (b) 3 wt\%.

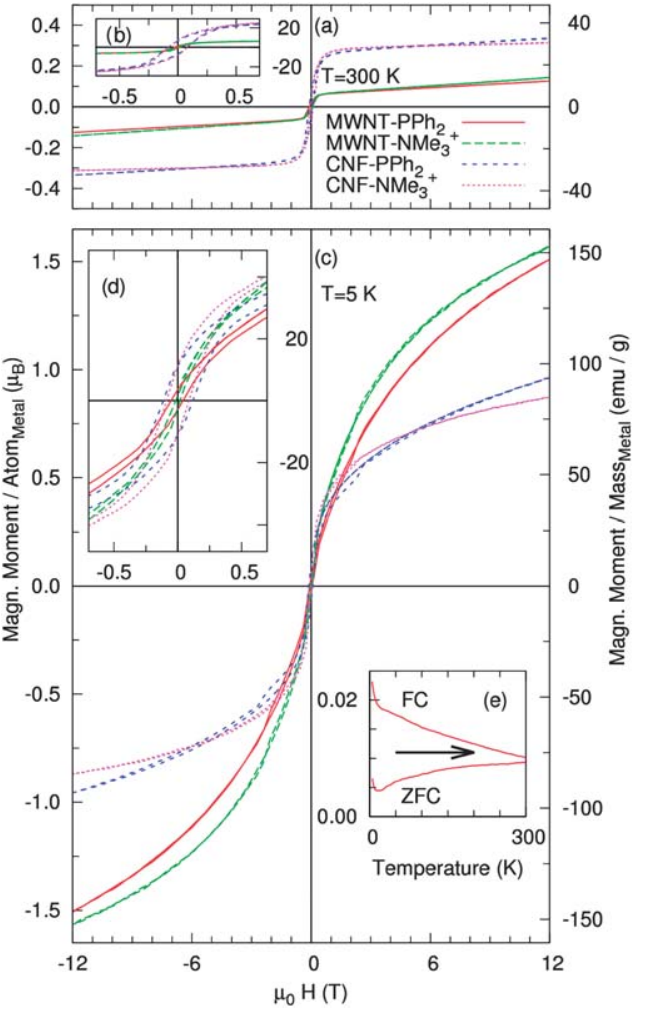

Fig. 11 (a) $M(\boldsymbol{H})$ hysteresis measurements at $300 \mathrm{~K}$ of the indicated samples prepared from $\left[\mathrm{HFeCO}_{3}(\mathrm{CO})_{12}\right]$ clusters between -12 and $12 \mathrm{~T}$; inset $(\mathrm{b})$ shows the low field region. (c) and inset (d) $M(\boldsymbol{H})$ hysteresis measurements at $5 \mathrm{~K}$. (e) Zerofield cooled and field cooled magnetization measured on heating with $\mu_{0} \boldsymbol{H}=$ $0.01 \mathrm{~T}$ for MWCNT-PPh 2 .

of the substrate, the MWCNT yielding smaller saturation magnetization and much smaller coercive field. The functionalization with $\mathrm{PPh}_{2}$ or $\mathrm{NMe}_{3}{ }^{+}$does not have a strong influence on the magnetic properties. The measurements carried out at 5 K, see Fig. 11(c) and (d), reveal that in addition to (blocked) superparamagnetic particles, the large amount of paramagnetic ions contribute to the magnetic response, as seen by the Langevin-like behavior superposed to the hysteretic and saturated behavior of the (blocked) superparamagnetic particles. The overall response is affected by the functionalization.
At least two different magnetic metal species are present in the samples, which can be distinguished from the hysteresis loops at different temperatures. The first species are blocked nanoparticles, likely Fe-Co. The blocking temperature above room temperature is in line with a possible domain formation, agglomeration, or core-shell structure in the nanoparticles, ${ }^{34}$ or with a possible oxidation of the nanoparticle shells. ${ }^{35}$ In order to clarify the origin of this behaviour, further structural investigations beyond the scope of this paper are needed. The second species is paramagnetic, most likely isolated metal ions, likely $\mathrm{Fe}($ III) or $\mathrm{Co}$ (II) not surrounded by other metals and which are not transformed to nanoparticles. The concentration amount of these species is related to the functionalized substrate. With this two component model, at room temperature, one expects to see mainly the nanoparticle contribution which quickly saturates and hides the weak 1/T paramagnetic response. At low temperature, for the nanoparticles, essentially the response is similar to that at room temperature, whereas the large paramagnetic response of the free atomic species is revealed at large fields.

A first approximation analysis of the paramagnetic Langevin response at low temperature, i.e. by taking into account only the high field part at $5 \mathrm{~K}$, yields estimations of the effective paramagnetic moments: for $\mathrm{PPh}_{2}$-functionalized carbons, $\mu_{\text {eff }}=$ 4.7(1) $\mu_{\mathrm{B}}$, while for $\mathrm{NMe}_{3}{ }^{+}$-functionalized carbons, $\mu_{\text {eff }}=5.9(1)$ $\mu_{\mathrm{B}}$. For high spin Fe(III) the moment is spin only, with $J=5 / 2$, and one expects $\mu_{\text {eff }}=5.92 \mu_{\mathrm{B}}$, which is in good agreement with the moment observed for the $\mathrm{NMe}_{3}{ }^{+}$functionalization. For high spin $\mathrm{Co}(\mathrm{II})$, the $L=3$ orbital moment is usually strongly quenched by the crystal field and the measured effective moment can vary, but for paramagnetic Co(II) salts $\mu_{\text {eff }}=4.8 \mu_{\mathrm{B}}$ is a typical value, ${ }^{36,37}$ very close to what is observed for the $\mathrm{PPh}_{2}$ functionalization. This shows that (isolated) ionic paramagnetism is present in our samples, with a strong dependence on the nature of functions present at the beginning. The ionic paramagnetic species are assumed to be stabilized by interactions with residues of organic functions. The $\mathrm{NMe}_{3}{ }^{+}$functionalization seems to lead to $\mathrm{Fe}(\mathrm{III})$ ions whereas the $\mathrm{PPh}_{2}$ group yields Co(II) ions. This is consistent with ion-paring occurring at the iron site and sequestering Fe from the cluster, and inversely $\mathrm{CO} /$ phosphine ligand exchange occurring on the cobalt part of 
the cluster. Summarizing, the functionalization affects the paramagnetic moment of the atoms that are not part of $\mathrm{Fe}-\mathrm{Co}$ particles, whereas the substrate strongly influences the amount of these atoms, as seen from a direct comparison of the relative saturation magnetization at 300 and $5 \mathrm{~K}$.

\section{Conclusions}

We have shown that CNF and MWCNT can be covalently functionalized with chelating phosphine or ammonium groups. All functionalization steps were characterized by elemental analysis, XPS, SEM or TEM to prove the success of the procedure. Textural characterization by nitrogen physisorption showed that the specific surface area decreased slightly. By combining the results obtained by elemental analysis and knowing the final specific surface area, the number of phosphine ligands present on the surface per unit of area could be calculated and corresponds to 1.5 and 0.45 anchoring sites per $\mathrm{nm}^{2}$ for CNF$\mathrm{PPh}_{2}$ and MWCNT-PPh${ }_{2}$, respectively.

The pristine, oxidized or functionalized supports were then used to graft two different iron-cobalt molecular clusters. Atomic absorption allowed us to confirm the incorporation of metals at the supports' surface. The samples were also characterized by SIMS. It can be concluded that before activation on phosphine or ammonium functionalized supports, the clusters were molecularly intact at the surface of the supports, as expected. However, in the case of oxidized or pristine supports, the typical cluster fragmentation pattern was not present anymore, which highlights definitely the advantage of using functionalized supports. No or little agglomeration was observed during thermal activation. The nanoparticles obtained after thermal activation were better dispersed on functionalized than on pristine support, proving that the surface functions act as stabilizing sites for the clusters. Magnetic characterization revealed a strong dependence of both the nature of the initial support and the nature of the functionalization arm on the observed magnetic properties. Blocked superparamagnetic FeCo nanoparticles were obtained in all cases, together with isolated paramagnetic ions. The obtained materials might find applications in heterogeneous catalysis and as building blocks for nanoelectronics and composite materials.

\section{Acknowledgements}

The authors gratefully acknowledge the Fonds National de la Recherche Scientifique (FNRS-FRS), the Interuniversity Attraction Pole Programme of the Belgian State (INANOMAT P6/17), the Fédération Wallonie-Bruxelles and the National Lottery (FRFC), and the Helmholtz Gemeinschaft Deutscher Forschungzentren (grant VH NG-407) for financial support. They also acknowledge the Fonds pour la formation à la Recherche dans l'Industrie et dans l'Agriculture (FRIA) for the research fellowship allotted to D.V. We are also grateful to P. Eloy and M. Genet for assistance with XPS in "pseudo in situ" mode, Prof. O. Riant and Charles Vriamont for organic chemistry developments, the Nanocyl company for supplying MWCNT, Applied
Sciences Inc. for supplying CNF and J.-F. Statsijns for technical support.

\section{Notes and references}

1 P. Serp, M. Corrias and P. Kalck, Appl. Catal., A, 2003, 253, 337.

2 N. Karousis, N. Tagmatarchis and D. Tasis, Chem. Rev., 2010, 110, 5366.

3 A. Rasheed, J. Y. Howe, M. D. Dadmun and P. F. Britt, Carbon, 2007, 45, 1072.

4 T. G. Ros, A. J. van Dillen, J. W. Geus and D. C. Koningsberger, Chem.-Eur. J., 2002, 8, 1151.

5 P. V. Laksminarayanan, H. Toghiani and C. U. Pittman Jr, Carbon, 2004, 42, 2433.

6 X. Li and M. R. Coleman, Carbon, 2008, 46, 1115.

7 C. Willocq, S. Hermans and M. Devillers, J. Phys. Chem. C, 2008, 112, 5533.

8 H. Paul, S. Basu, S. Bhaduri and G. K. Lahiri, J. Organomet. Chem., 2004, 689, 309.

9 A. Indra, S. Basu, D. G. Kulkarni, C. S. Gopinath, S. Bhaduri and G. K. Lahiri, Appl. Catal., A, 2008, 344, 124.

10 X. Hou, L. Wang, F. Zhou and F. Wang, Carbon, 2009, 47, 1209.

11 A. Suri, A. K. Chakraborty and K. S. Coleman, Chem. Mater., 2008, 20, 1705.

12 R. Paul, P. Kumbhakar and A. K. Mitra, Appl. Surf. Sci., 2011, 257, 6699.

13 C. E. Hamilton, D. Ogrin, L. Mcjilton, V. C. Moore, R. Anderson, R. E. Smalley and R. Barron, Dalton Trans., 2008, 2937.

14 C. E. Hamilton and A. R. Barron, Main Group Chem., 2009, 8, 275.

15 G. Hogarth, S. E. Kabir and E. Nordlander, Dalton Trans., 2010, 39, 6153.

16 C. Femoni, M. C. Iapalucci, F. Kasmalder, G. Longoni and S. Zacchini, Coord. Chem. Rev., 2006, 250, 1580.

17 Metal Clusters in Chemistry, ed. P. Braustein, L. A. Oro and P. R. Raithby, Wiley-VCH, Weinheim, 1999.

18 A. Siani, B. Captain, R. D. Adams, O. S. Alexeev and M. D. Amiridis, Top. Catal., 2011, 54, 318.

19 S. Hermans, C. Diverchy, O. Demoulin, V. Dubois, E. Gaigneaux and M. Devillers, J. Catal., 2006, 243, 239.

20 P. Chini, L. Colli and M. Peraldo, Gazz. Chim. Ital., 1960, 90, 1005.

21 R. S. Armstrong, T. Bell, A. F. Master and M. A William, Polyhedron, 1990, 9, 2815.

22 P. Bertrand and L.-T. Weng, Mikrochim. Acta, 1996, 13, 167.

23 J. Li, M. J. Vergne, E. D. Mowles, W. H. Zhong, D. M. Hercules and C. M. Lukehart, Carbon, 2005, 43, 2883.

24 C. U. Pittman, G. R. He, B. Wu and S. D. Gardner, Carbon, 1997, 35, 317.

25 C. Willocq, D. Vidick, B. Tinant, A. Delcorte, P. Bertrand, M. Devillers and S. Hermans, Eur.J. Inorg. Chem., 2011, 4721.

26 V. Bruyr, M. Devillers and S. Hermans, J. Mater. Chem., 2012, 22, 14479. 
27 Y.-H. Li, S. Wang, Z. Luan, J. Ding, C. Xu and D. Wu, Carbon, 2003, 41, 1057.

28 C.-H. Li, K.-F. Yao and J. Liang, Carbon, 2003, 41, C01.

29 S. Kundu, Y. Wang, W. Xia and M. Muhler, J. Phys. Chem. C, 2008, 112, 16869.

30 U. Dettlaff-Weglikowska, V. Skakalova, R. Graupner, S. H. Jhang, B. H. Kim, H. J. Lee, L. Ley, Y. W. Park, S. Berber, D. Tomanek and S. Roth, J. Am. Chem. Soc., 2005, 127, 5125.

31 J. K. Wassei, K. C. Cha, V. C. Tung, Y. Yang and R. B. Kaner, J. Mater. Chem., 2011, 21, 3391.

32 C. Laslau, W. Henderson, Z. D. Zujovic and J. Travas-Sejdic, Synth. Met., 2010, 160, 1173.
33 R. S. Armstrong, T. Bell, A. L. Chaffee, V. W. L. Chin, H. J. Loeh, A. B. J. Lucchese, A. F. Masters and M. A. Williams, Appl. Catal., 1989, 47, 243.

34 A. Douvalis, R. Zboril, A. Bourlinos, J. Tucek, S. Spyridi and T. Bakas, J. Nanopart. Res., 2012, 14, 1130.

35 J. Noguésa, J. Sorta, V. Langlaisb, V. Skumryeva, S. Suriñachb, J. S. Muñozb and M. D. Barób, Phys. Rep., 2005, 422, 65.

36 K. H. J. Buschow and F. R. De Boer, Physics of Magnetism and Magnetic Materials, Kluwer Academic, New York, 2003.

37 S. Blundell, Magnetism in Condensed Matter, Oxford Master Series in Condensed Matter Physics, Oxford University Press, Oxford, 2001. 